\title{
A study on knowledge, attitude and practices among parents towards children with strabismus in Goa
}

\author{
J A Cacodcar', Tanvi Poy Raiturcar, ${ }^{2, *}$, Ugam Usgaonkar ${ }^{3}$, Marushka Aguiar ${ }^{4}$ \\ ${ }^{\mathbf{1 , 3}}$ Professor and HOD, ${ }^{2}$ Senior Resident, ${ }^{\mathbf{4}}$ Junior Resident, ${ }^{1}$ Dept. of Preventive and Social Medicine, ${ }^{\mathbf{2}, 3,4}$ Dept. of Ophthalmology, \\ Goa Medial College, Goa, India \\ *Corresponding Author: Tanvi Poy Raiturcar \\ Email: tanvi1491@gmail.com
}

\begin{abstract}
Context: Strabismus is one of the most common ocular morbidities affecting children, with a prevalence of 3 to $5 \%$ among children. Early diagnosis and treatment is very essential for successful treatment, which is only possible if the parents notice the signs and symptoms of strabismus early and seek timely treatment from qualified Ophthalmologists.

Aims: To assess the level of knowledge, attitude \& practices among parents towards children suffering from strabismus in Goa.

Settings and Design: Cross-sectional study.

Materials and Methods: 75 parents of children with strabismus and aged between 6 months to 14 years were administered a pretested proforma containing 12 questions, which included questions to assess their knowledge about strabismus and their attitudes and practices towards the condition, as well as their educational qualifications.

Statistical Analysis Used: Percentages and proportions.

Results: $90.66 \%$ had noticed the deviation of the eyes on their own. $81.33 \%$ were bothered by their child's strabismus. $74.66 \%$ reported that other people notice their child's strabismus. $76 \%$ felt uncomfortable when asked about their child's strabismus. $53.33 \%$ were aware that strabismus may come in the way of their child's scholastic performance and $44 \%$ feared fewer opportunities at school. $44 \%$ felt that their child has difficulty in socialising. $65.33 \%$ were aware about its effects on their child's vision. $48 \%$ attributed psychological effects in their child due to strabismus. $56 \%$ were aware that strabismus is treatable.

Conclusion: Hence health education is very important to increase the awareness and change myths about strabismus; thus making early diagnosis and successful treatment possible.
\end{abstract}

Keywords: Goa, KAP, Parents, Strabismus.

\section{Introduction}

Strabismus is one of the most common ocular morbidities affecting children, with a prevalence of 3 to $5 \%$ among children. ${ }^{1,2}$ The problems associated with strabismus include diplopia, asthenopia, decreased vision, poor development of binocular single vision, stereopsis, and cosmetic blemish.

Apart from this, affected children may develop psychological problems such as poor self-esteem and self-confidence, difficulty in adjustment and making new friends, other behavioural \& psychosocial problems and poor scholastic performance. ${ }^{3}$

The success of interventions for strabismus primarily depends upon on the age of presentation. Hence its early diagnosis and treatment is very essential. The treatment modalities include use of corrective spectacles, prisms, orthoptic exercises, amblyopia therapy and surgery. Early diagnosis and treatment is only possible if the parents notice the signs and symptoms of strabismus early and seek timely treatment from qualified Ophthalmologists.

In this study we have tried to assess the level of knowledge, attitude \& practices among parents towards children suffering from strabismus in Goa.

\section{Materials and Methods}

A cross-sectional study was conducted at the department of Ophthalmology, Goa Medical College and Hospital which is a tertiary care hospital in Goa between January to May 2018, to assess the knowledge, attitude and practices regarding strabismus among parents of children suffering from strabismus; after obtaining approval from the Institutional Ethical Committee at Goa Medical College and Hospital.

A total of 75 parents of children aged between 6 months to 14 years were included in the study. They were assured confidentiality, and after an informed consent, they were administered a pretested proforma containing 12 close ended questions, which included questions to assess their knowledge about strabismus and their attitudes and practices towards the condition in their child. The educational qualifications of the participants were also studied and noted in the questionnaire.

The data thus collected was entered in Microsoft Excel 2010 version and the statistical tests used for analysis included percentages and simple proportions.

\section{Results}

Out of the 75 parents who participated in our study, majority i.e. $68(90.66 \%)$ had noticed the deviation of the eyes on their own. Only 7 (9.34\%) were made aware of the condition by friends, relatives or teachers.

Most of the participants i.e. $61(81.33 \%)$ parents were bothered by their child's strabismus. 56 (74.66\%) parents reported that other people notice their child's strabismus. 57 (76\%) felt uncomfortable when people asked them about the eye condition of their child. 
$40(53.33 \%)$ parents had knowledge about the fact that strabismus may come in the way of their child's performance in school and $33(44 \%)$ felt that their children may have fewer opportunities at school because of the condition.

$33(44 \%)$ felt that their child has difficulty in making friends because of strabismus. Though 49 $(65.33 \%)$ were aware that strabismus affects their child's vision, a significant $26(34.67 \%)$ were ignorant that strabismus affected their child's vision.

$36(48 \%)$ parents attributed psychological effects in their child due to strabismus, which included difficulty in self-image, poor interpersonal relationships and lowered self-confidence.

$42(56 \%)$ parents were aware that strabismus is treatable with early intervention, whereas a significant $33(44 \%)$ of them were unaware that strabismus is a treatable condition.

\section{Discussion}

Out of the 75 parents who participated in our study, $65 \%$ had completed graduation or higher education, while $35 \%$ were either uneducated or had completed education less than graduation.

The knowledge and attitude of the parents towards strabismus was comparable to their level of education, as the better educated had greater knowledge about their child's eye condition.

Majority i.e. 20 out of 26 parents from the lower educated group $(76.92 \%)$, had not noticed their child's eye condition on their own, and were made aware of the same by friends, relatives, or school teachers. These were the children who presented much later to an Ophthalmologist; and some of them i.e. 5 children had already developed strabismic amblyopia, making the treatment difficult.

Parents belonging to the lower educated group were more bothered about the cosmetic appearance. These parents were uncomfortable when other people asked about their child's condition. They were of the opinion that their child will have fewer opportunities in school because of their eye condition, and also face difficulties in making friends. They had a negative impression about their child's condition, and a higher level of stress as compared to the higher educated group of parents.

Almost half the parents (44\%) were unaware that strabismus could be treated and had accepted the condition.

37 out of 49 parents belonging to the higher educated group $(75.51 \%)$ had noticed the child's eye condition themselves, and presented much earlier to the Ophthalmologist, making treatment more successful. They also had a higher level of knowledge and awareness about strabismus, and how it can affect the child's vision. They had a positive attitude, and many of them were not uncomfortable in discussing the condition with other people when asked. They did not feel that their child will have lesser opportunities at school because of strabismus, or difficulty in making new friends. Such parents were also aware that the condition is treatable with timely intervention.

Our study finding is similar to another study by Singh et $\mathrm{al}^{4}$ in which they found a better awareness about strabismus in parents who had higher education compared to the less educated group, and that the source of information for the educated was mainly the internet or discussion with an Ophthalmologist, which was more reliable as compared to information from relatives and friends who contributed the main source of information to the less educated parents.

In our study $48 \%$ parents reported signs of psychological effects due to strabismus, as compared to $36.67 \%$ reported in a study by Singh et al. ${ }^{4}$ Such children may develop problems with adjustment, difficulty in making new friends, and have a lower selfesteem. In another study Satterfield et $\mathrm{al}^{3}$ reported that strabismus had a negative impact on the quality of life of children, and led to poor scholastic performance. It is often seen that children with strabismus are ridiculed by other children in school because of their eye condition, and this further worsens the psychological effects and self-esteem. The children may face anxiety and depression because of their eye condition.

It is also seen that parents are also constantly troubled by their child's eye condition and are under stress. Many parents are very uncomfortable to speak about the condition to others when questioned, and do not like it when others notice the squinting in their child. This may cause them to face difficulties with parenting, and psychosocial effects in the parents themselves, such as depression and anxiety. Such parents often have a sense of guilt and this affects their family relationships and interaction with their child. From our study we have been able to conclude that the level of stress is higher among the lower educated group of parents as compared to the higher educated group of parents, due to differences in the level of knowledge about the condition and awareness about its treatable nature. Our finding is similar to that reported by Singh et $\mathrm{al}^{4}$ in their study where the higher educated group of parents were less stressed about their child's eye condition as compared to the lower educated group of parents.

The first step of treatment of strabismus is to detect and correct any refractive error that is present in the child. This is achieved by using lenses or prisms. Where the fusional reserves are low, the child may be advised orthoptic treatment in the form of fusional exercises to be done at home, with anti-supression measures when required, to achieve alignment of the visual axes of the two eyes. Detection and prevention of amblyopia is a very important aspect of successful management of strabismus. Only when the refractive error is completely corrected and amblyopia is treated, the child is to be posted for surgery. A complete 
systemic evaluation of the child is also very important to rule out systemic illnesses such as thyroid dysfunction and to look for any underlying neurological disturbance. The main goals of strabismus surgery are two i.e. cosmetic correction and functional improvement. This means that there should be a satisfactory alignment of the two visual axes in all nine cardinal positions of gaze and to provide and maintain a good visual acuity and binocular single vision in both the eyes, with preservation of stereo acuity and field of vision. $^{5,6}$ The strabismologist should also make it a point to explain to the parents that there is always a chance that the child mare require a second surgery if the outcome of the first surgery is not satisfactory.

It is a fact that early diagnosis and treatment of the eye condition enables them to have a better psychosocial development and prevent behavioural and adjustment problems. Studies done in the past by Menon et al, ${ }^{7}$ Ziaei et al, ${ }^{8}$ Archer SM et al, ${ }^{9}$ and Koklanis $\mathrm{K}$ et $\mathrm{al}^{10}$ have showed that there is a positive change in pyscho-social function in children after successful strabismus treatment. Children who received successful treatment developed better self- image, higher self-confidence, better interpersonal relationships, and also improved academic performance and performance at sports.

The parent's knowledge and attitudes toward strabismus in their child is a very important aspect of strabismus therapy. This is because the time at which the parents notice the strabismus in their child and seek treatment from an Ophthalmologist is very important. The earlier the treatment is started, the better are the results. As the age advances there is diminution in vision, reduction in field of vision, restriction of ocular motility, diplopia, loss of stereopsis and amblyopia. Also the parents should be in a position to understand the nature of treatment and practice them at home; such as occlusion, orthopic exercises, constant use of spectacles or prisms. Explaining to the parents regarding strabismus and removing myths about the same is hence very important and can only be achieved by way of health education; so as to improve awareness about strabismus.

Table 1: Knowledge, attitude and practices among Goan parents towards their children with strabismus

\begin{tabular}{|l|c|c|}
\hline \multicolumn{1}{|c|}{ Study variables } & Yes Number (\%) & No Number (\%) \\
\hline $\begin{array}{l}\text { Parents who noticed strabismus in their child on their } \\
\text { own }\end{array}$ & $68(90.66 \%)$ & $7(9.34 \%)$ \\
\hline Parents who felt bothered by their child's strabismus & $61(81.33 \%)$ & $14(18.67 \%)$ \\
\hline $\begin{array}{l}\text { Parents who reported that other people noticed the } \\
\text { strabismus in their child }\end{array}$ & $56(74.66 \%)$ & $19(25.34 \%)$ \\
\hline $\begin{array}{l}\text { Parents who felt strabismus hindered their child's } \\
\text { scholastic performance }\end{array}$ & $40(53.33 \%)$ & $35(46.67 \%)$ \\
\hline $\begin{array}{l}\text { Parents who felt that their child would have lesser } \\
\text { opportunities at school due to strabismus }\end{array}$ & $33(44 \%)$ & $42(56 \%)$ \\
\hline $\begin{array}{l}\text { Parents who felt their child has difficulties in making } \\
\text { friends due to their condition }\end{array}$ & $33(44 \%)$ & $42(56 \%)$ \\
\hline $\begin{array}{l}\text { Parents who felt uncomfortable upon being asked by } \\
\text { others about their child's condition }\end{array}$ & $57(76 \%)$ & $18(24 \%)$ \\
\hline $\begin{array}{l}\text { Parents who felt that strabismus hindered their child's } \\
\text { vision }\end{array}$ & $49(65.33 \%)$ & $26(34.67 \%)$ \\
\hline $\begin{array}{l}\text { Parents reporting difficulty in reading by their child } \\
\text { with strabismus }\end{array}$ & $43(57.33 \%)$ & $32(42.67 \%)$ \\
\hline $\begin{array}{l}\text { Parents reporting that their affected child closes one eye } \\
\text { while reading }\end{array}$ & $28(37.33 \%)$ & $47(62.67 \%)$ \\
\hline $\begin{array}{l}\text { Parents who attributed psychological effects in their } \\
\text { child due to the strabismus }\end{array}$ & $36(48 \%)$ & $39(52 \%)$ \\
\hline $\begin{array}{l}\text { Parents aware about treatable nature of strabismus in } \\
\text { their child }\end{array}$ & $42(56 \%)$ & $33(44 \%)$ \\
\hline
\end{tabular}

\section{Conclusion}

The success of strabismus treatment primarily depends on the age of presentation, when early diagnosis and treatment is possible. This is only possible with better awareness about strabismus among parents. Lack of knowledge about strabismus delays the entire process of identification and treatment of strabismus. Hence health education is very important to increase the awareness and change myths about strabismus; thus making early diagnosis and successful treatment possible. Early diagnosis and treatment will also help prevent psychosocial problems and behavioural disorders among children affected with strabismus and improve academic performance and also prevent the development of amblyopia. 


\section{References}

1. Donelly UM, Stewart NM, Hollinger M. Prevalence and outcomes of childhood visual disorders. Ophthalmic Epidemiol. 2005;12:243-50.

2. Graham PA. Epidemiology of strabismus. $\mathrm{Br} \mathrm{J}$ Ophthalmol. 1974;58:224-31.

3. Satterfield D, Keltner JL, Morrison TL. Psychosocial aspects of strabismus study. Arch Ophthalmol. 1993;111:1100-5.

4. Singh A, Rana V, Patyal S, Kumar S, Mishra SK, Sharma VK. To assess knowledge and attitude of parents towards children suffering from strabismus in the Indian subcontinent. Indian J Ophthalmol. 2017;65:603-6.

5. Kushner BJ, Fisher MR, Lucchese NJ, Morton GV. Factors influencing response to strabismus surgery. Arch Ophthalmol. 1993;111:75-9.

6. Abbasoglu OE, Sener EC, SanacAS. Factors influencing the successful outcome and response in strabismus surgery. Eye Lond. 1996;10:315-20.

7. Menon V, Saha J, Tandon R, Mehta M, Khokhar S. Study of psychosocial aspects of strabismus. J Pediatr Ophthalmol Strabismus. 2002;39:203-8.
8. Ziaei H, Katibeh M, Mohammadi S, Mirzaei M, Moein HR, Kheiri B, et al. The impact of strabismus surgery on quality of life in children. $J$ Ophthalmic Vis Res. 2016;11:188-92.

9. Archer SM, Musch DC, Wren PA, Guire Ke, Del Monte MA. Social and emotional impact of strabismus surgery on quality of life in children. J AAPOS. 2005;9:148-51.

10. Koklanis K, Abel LA, Aroni R. Psychosocial impact of amblyopia and its treatment: A multidisciplinary study. Clin Exp Ophthalmol. 2006;34:743-50.

How to cite this article: Cacodcar J. A, Raiturcar T. P, Usgaonkar U, Aguiar M. A study on knowledge, attitude and practices among parents towards children with strabismus in goa. Indian J Clin Exp Ophthalmol. 2018;4(4):507510. 